\title{
Molecular Probe of Pairing in the BEC-BCS Crossover
}

\author{
G. B. Partridge, K. E. Strecker $*$ R. I. Kamar, M. W. Jack, and R. G. Hulet \\ Department of Physics and Astronomy and Rice Quantum Institute, Rice University, Houston, Texas 77251
}

(Dated: April 27, 2018)

\begin{abstract}
We have used optical molecular spectroscopy to probe the many-body state of paired ${ }^{6} \mathrm{Li}$ atoms near a broad Feshbach resonance. The optical probe projects pairs of atoms onto a vibrational level of an excited molecule. The rate of excitation enables a precise measurement of the closed-channel contribution to the paired state. This contribution is found to be quite small, supporting the concept of universality for the description of broad Feshbach resonances. The dynamics of the excitation provide clear evidence for pairing across the BEC-BCS crossover, and into the weakly interacting BCS regime.
\end{abstract}

PACS numbers: 03.75.Ss, 33.80.Ps, 67.90.+z, 03.75.Nt

Feshbach resonances were recently used to explore the possibility of superfluidity and Cooper pairing in atomic Fermi gases of ${ }^{40} \mathrm{~K}$ and ${ }^{6} \mathrm{Li}$. Two experiments have produced evidence for correlated pairs [1], while others report evidence for superfluidity [2]. These experiments are noteworthy because they are performed on the highfield side of the resonance, where the $s$-wave scattering length $a$ is negative and, according to two-body physics, diatomic molecules associated with the resonance are energetically unstable. These intriguing experiments raise important questions regarding the nature of Feshbachinduced pairs, including their relation to bound molecules and the role played by many-body effects.

Atomic Feshbach resonances involve a collision of a pair of atoms in an open channel coupled to a bound molecular state of a closed channel [3]. In the broad ${ }^{6} \mathrm{Li}$ resonance, the open channel corresponds to two atoms interacting mainly via the electronic spin-triplet potential, while the closed-channel is predominantly spin-singlet. Two-body theory predicts the formation of a weaklybound molecular state on the "BEC" side resonance, where $a>0$. These "dressed" molecules are superpositions of open-channel atoms with the "bare" molecules of the closed channel. For resonances that are broad compared to the Fermi energy, the closed channel character of the dressed molecules is expected to be small throughout an experimentally relevant region about the resonance 4]. In this case, the resonance may be well-described by a single-channel model where the physics is universal, such that the macroscopic properties of the superfluid are independent of the microscopic physics that underlie the two-body interactions. Universality in the description of pairing in atomic gases establishes their relevance to other systems, most notably high-temperature superconductors.

In the experiment reported here, a laser is used to project the dressed molecules/pairs onto an excited singlet molecular state. By starting with an evaporatively cooled gas on the BEC side of the resonance, followed by an adiabatic change in the magnetic field, a nearly zero temperature gas can be probed throughout the BEC-
BCS crossover. This enables a direct measurement of the closed channel fraction and, for the first time in an atomic gas, provides clear evidence for the presence of pair correlations in the weakly interacting BCS regime.

Our apparatus and methods for producing strongly interacting Fermi gases of ${ }^{6} \mathrm{Li}$ atoms have been described previously [5]. The atoms are confined in a single-beam optical trap which, at full laser intensity, has a trap depth of $25 \mu \mathrm{K}$ and radial and axial freqencies of $\nu_{r}=2270 \mathrm{~Hz}$ and $\nu_{z}=21 \mathrm{~Hz}$, respectively. Curvature in the magnetic bias field modifies the axial frequency such that $\nu_{z}^{\prime}=\sqrt{\nu_{z}^{2}+\lambda B}$, where $\lambda=.029(3) \mathrm{Hz}^{2} / \mathrm{G}$. An incoherent equal mixture of the two lowest going Zeeman sublevels $\left(F=1 / 2, m_{F}= \pm 1 / 2\right)$ is created [5]. For the magnetic fields of interest $(B \geq 600 \mathrm{G})$ these states are nearly electronically spin polarized, but differ in their nuclear spin projections. They interact strongly via a broad Feshbach resonance located near 834 G [6, 7].

A molecular BEC is created as in other ${ }^{6} \mathrm{Li}$ experiments 8]. We prepare the spin mixture at $754 \mathrm{G}$ (corresponding to $a \simeq 3680 a_{o}[9]$ ), and dressed molecules form through three-body recombination. The molecules are evaporatively cooled by reducing the trap depth in $750 \mathrm{~ms}$ in an approximately exponential trajectory. Their distribution is imaged in the trap with the same optical probe used to image the atoms. Figure 1 shows that the condensate fraction can be controlled by the final trap depth. The crossover regime is probed by preparing the gas at $754 \mathrm{G}$ and adiabatically changing the magnetic field to any desired final value. We find that adiabatic field excursions, even those going across the resonance, result in no detectable heating upon returning to the original field [10].

A molecular probe laser drives transitions between the dressed molecules/pairs and an electronically excited molecular singlet level. These transitions result in spontaneous emission and a detectable loss of atoms from the trap. We chose to drive transitions to the $v^{\prime}=68$ level of the $A^{1} \Sigma_{u}^{+}$excited state because it has the largest FranckCondon wavefunction overlap (0.077) with the resonant channel bare molecule, the $X^{1} \Sigma_{g}^{+}(v=38)$ level. The 


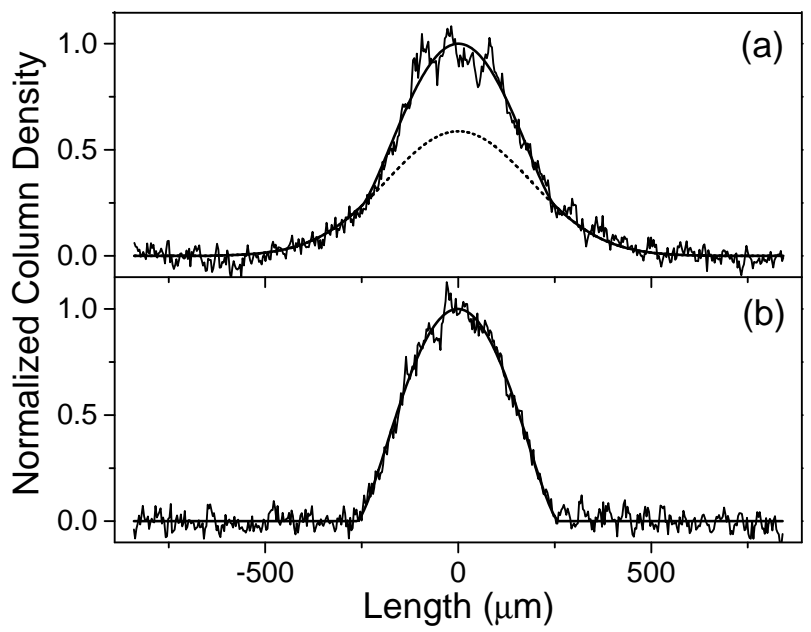

FIG. 1: In-situ absorption image profiles showing a molecular BEC. These images were recorded at a field of $695 \mathrm{G}$ after evaporation at $754 \mathrm{G}$. For (a), the optical trap depth was lowered to $0.5 \mu \mathrm{K}$. The solid line is a fit to a Gaussian (dotted line) plus Thomas-Fermi distribution which distinguishes the condensate from residual thermal molecules. For (b), the trap depth is reduced to $0.27 \mu \mathrm{K}$, producing an essentially pure molecular condensate with the number of molecules, $N=$ 46, 000. We estimate that the condensate fraction is $>90 \%$, implying $T / T_{c} \leq 0.5$, where $T_{c}$ is the critical temperature for BEC at $695 \mathrm{G}$. The solid line is a Thomas-Fermi distribution.

$v^{\prime}=68$ level has a classical turning point of $36 a_{0}$. A quantity $Z$ is defined in terms of $\Gamma$, the rate of photoexcitation, by $\Gamma=Z \Omega^{2} / \gamma$, where $\Omega=\left\langle\psi_{v^{\prime}=68}(S=\right.$ $\left.0)\left|\vec{d} \cdot \overrightarrow{E_{\mathrm{L}}}\right| \psi_{v=38}(S=0)\right\rangle$ is the on-resonance Rabi frequency, $\vec{d}$ is the transition dipole, $\vec{E}_{\mathrm{L}}$ is the laser field of the molecular probe, and $\gamma=(2 \pi) 11.7 \mathrm{MHz}$ is the linewidth of the excited molecular state [11]. The dressed molecules/pairs can be expressed as a superposition of the $v=38$ singlet molecules and free atom pairs in the triplet channel $[\underline{3}]$ :

$$
\left|\psi_{\mathrm{p}}\right\rangle=Z^{1 / 2}\left|\psi_{v=38}(S=0)\right\rangle+(1-Z)^{1 / 2}\left|\phi_{\mathrm{a}}(S=1)\right\rangle,
$$

and $Z$ can be identified as the component fraction of the dressed molecules in the closed-channel. For a typical probe laser intensity of $20 \mathrm{~mW} / \mathrm{cm}^{2}, \Omega=(2 \pi) 2.6 \mathrm{MHz}$ 11.

$Z$ is measured at various fields by the following process: first, the molecular gas is prepared at $754 \mathrm{G}$. The field is then linearly ramped to a new value $B$ in $160 \mathrm{~ms}$ and held for $20 \mathrm{~ms}$ before the molecular probe is pulsed on for a fixed duration. The probe removes atoms at a rate $\Gamma$ determined by $Z$. The field is then linearly returned to $754 \mathrm{G}$ in $160 \mathrm{~ms}$, where the number of remaining atoms is determined by optical absorption imaging. The measured numbers are normalized to data obtained by the same procedure, but without firing the molecular probe.

Figure 2] shows the normalized loss of atoms at $B=695$

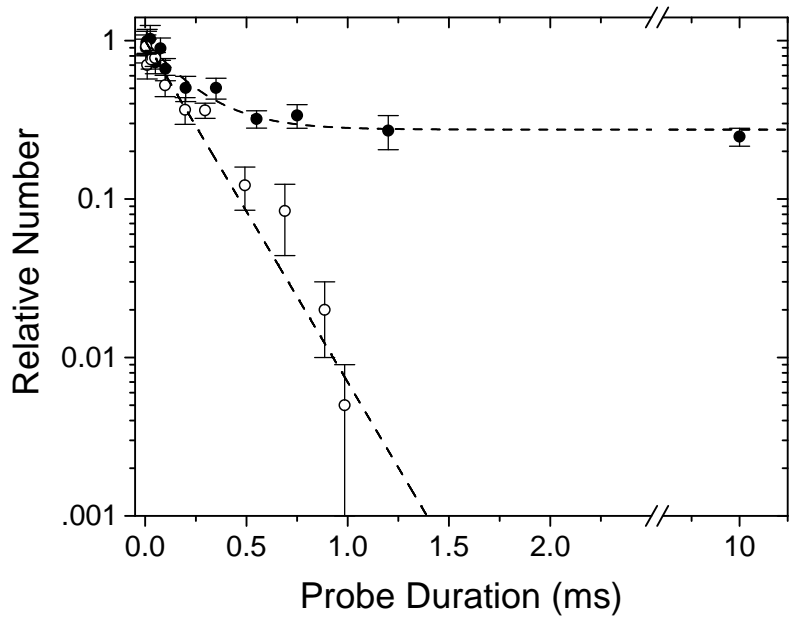

FIG. 2: Loss of signal vs. molecular probe duration at 695 G. The open circles correspond to a gas evaporatively cooled to a nearly pure molecular BEC, while the closed circles correspond to full trap depth, where $T / T_{F} \simeq 0.75$. The dashed lines are fits to exponentials, with a leftover fraction of $25 \%$ in the high temperature case. The time axis for the BEC data was scaled to account for differences in molecular probe laser intensity between the BEC data $\left(30 \mathrm{~mW} / \mathrm{cm}^{2}\right)$ and the high temperature data $\left(15 \mathrm{~mW} / \mathrm{cm}^{2}\right)$. We have verified that the loss rate depends linearly on intensity. The error bars represent the statistical standard deviation from the mean of $\sim 10$ independent measurements.

$\mathrm{G}$ as a function of the molecular probe duration for two different temperatures. In the case of the pure condensate, the gas is well into the BEC regime, since $a \simeq 1510 a_{o}$ and $k_{F} a \simeq 0.15$. As expected in this case, the entire trap can be depleted for sufficiently long probe duration. Furthermore, the signal decays exponentially, indicative of a one-body loss process. For the higher temperature data, the loss is initially exponential, but $25 \%$ of the initial number remain after a long probe duration. The remainder can be understood as the dissociated atom fraction, which is determined by the fitted temperature $T=1.8 \mu K$ and the calculated binding energy $E_{b}=13$ $\mu \mathrm{K}$ of the dressed molecules. A detailed balance calculation gives approximate agreement with the observed dissociated fraction. The probe only weakly couples to free atoms since they have a relatively small singlet character and excitation occurs only by two-body photoassociation.

Figure 3 shows the loss of signal vs. probe duration at $865 \mathrm{G}$, where $a \simeq-15600 a_{o}$. At this field, the gas is in the strongly interacting regime where $k_{F}|a|>1$. According to two-body physics, there are no bound states above the resonance at $834 \mathrm{G}$, and probe-induced loss would arise exclusively from two-body photoassociation. Nonetheless, the observed loss for the gas prepared in a nearly pure BEC fits to an exponential rather than to a two-body process. At full trap depth, the decay consists 


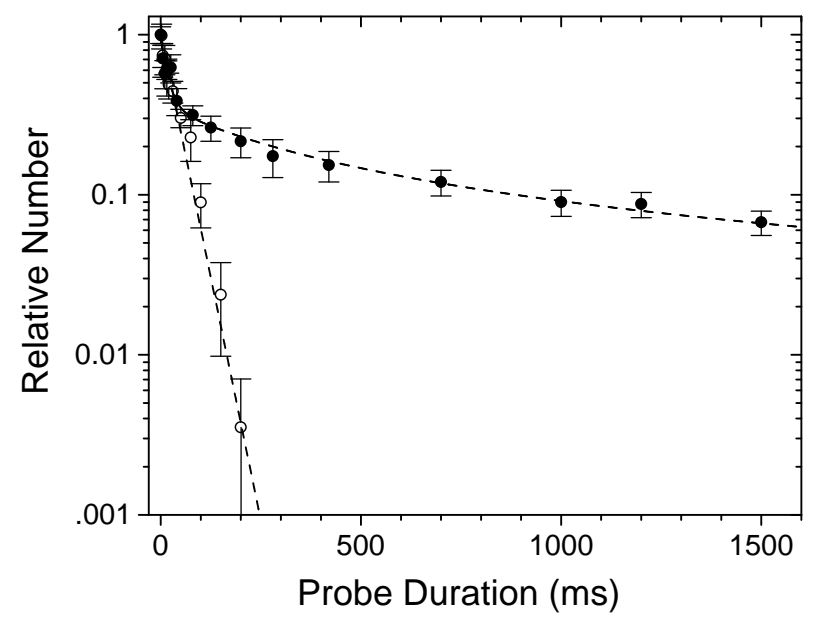

FIG. 3: Same as for Fig. 2 except at 865 G. The dashed line in the case of the full trap depth data (closed circles) is a fit to a "two-fluid" model where one component decays via a rapid one-body loss process and the other via a slower twobody loss process. Approximately $75 \%$ of the gas is lost by the initial fast process.

of two parts: an initial exponential decay, followed by a much slower two-body process. As the temperature of the cloud is $T \simeq 0.75 T_{F}$, this initial fast decay may indicate the presence of uncondensed paired fermions [13] or finite-lifetime molecules. The slower (two-body) process is ascribed to free-bound photoassociation, which is supported by the fact that the extracted two-body rate coefficient, $K_{2}=4.9(3.3) \times 10^{-10}\left(\mathrm{~cm}^{3} \mathrm{~s}^{-1}\right) /\left(\mathrm{W} \mathrm{cm}^{-2}\right)$, agrees well with the calculated value of $9.8(2.6) \times 10^{-10}$ $\left(\mathrm{cm}^{3} \mathrm{~s}^{-1}\right) /\left(\mathrm{W} \mathrm{cm}^{-2}\right)$ obtained using the expression for $K_{2}$ given in Ref. [1], where the uncertainties arise mainly from the temperature determination.

Figure 4 shows the extracted values of $Z$ for fields between 600 and $920 \mathrm{G}$. Below $600 \mathrm{G}$, the dressed molecule lifetime is too short to obtain a reliable measurement of $Z$. Also shown in Figure 4 are the results of a coupled channels calculation, which represents an exact two-body theory. An analytic expression for $Z$ on the BEC side of the resonance has been given in Ref. [14] and is in good agreement with our calculation. While the two-body theory accurately represents the data for fields below resonance, there is complete disagreement above resonance. Two-body theory predicts that $Z$ goes to zero as the resonance is approached, since the size of the dressed molecules diverges at resonance and produces a vanishing overlap with the excited molecules. The measured quantity, however, continues smoothly through resonance, decreasing exponentially with increasing field. Although the closed-channel fraction is finite and measurable, its magnitude above resonance is sufficiently small, $\lesssim 10^{-5}$, that the expectation of the number of closed-channel molecules is less than one.

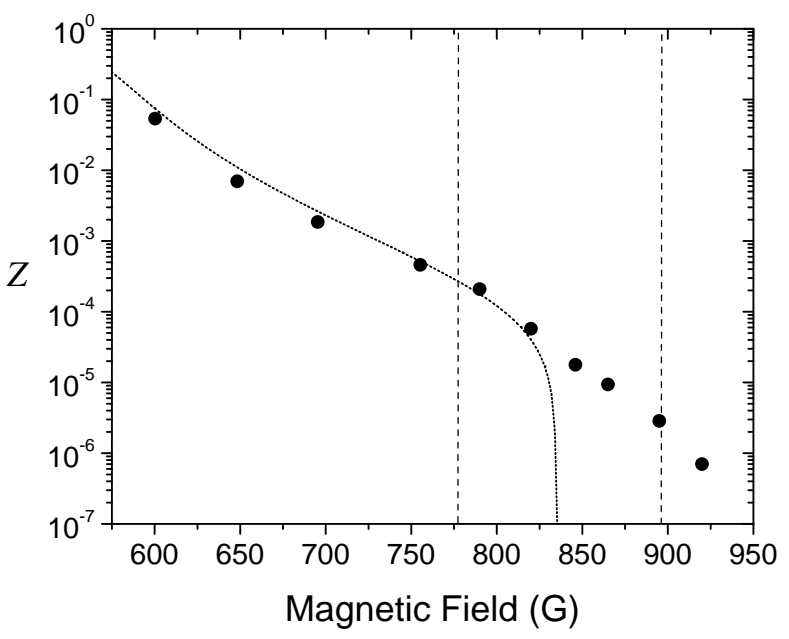

FIG. $4: Z$ vs. $B$. The closed circles represent the value of $Z$ extracted from measured values of $\Gamma$. In the case of the $920 \mathrm{G}$ point, the loss is not exponential, but the value of $\Gamma$ is taken to be the initial loss rate. The uncertainty in $Z$ is approximately equal to the size of the closed circles, and is due mainly to uncertainty in the probe laser intensity. The dotted line shows a comparison with results obtained from a coupled channels calculation [15]. The vertical dashed lines represent the boundaries of the strongly-interacting regime, $k_{F}|a|>1$, where $k_{F}$ is evaluated using typical values of $N$ at the low and high field extremes. Although shot-to-shot variations in $N$ are $30 \%$, the average value of $N$ at each field is between 13,000 and 90,000 due to day to day variations. $T_{F}$ is between 200 and $600 \mathrm{nK}$ due to differences in $N$ as well as the trap frequencies. For all the data, $T<T_{c}$ and for the points above $850 \mathrm{G}, T<0.5 T_{c}$, where $T_{c}$ refers to the critical temperature at $695 \mathrm{G}$. The gas is expected to be adiabatically cooled by the increasing field ramp for fields above $755 \mathrm{G}$ [16].

The small closed channel fraction suggests comparison with a single channel model. We note that $\Gamma$ is proportional to the local pair correlation function $G_{2}(r, r)=\left\langle\hat{\psi}_{\downarrow}^{\dagger}(r) \hat{\psi}_{\uparrow}^{\dagger}(r) \hat{\psi}_{\uparrow}(r) \hat{\psi}_{\downarrow}(r)\right\rangle$, where $\hat{\psi}_{\uparrow}$ and $\hat{\psi}_{\downarrow}$ are the fermionic field operators for atoms in different internal states. In the mean-field approximation $G_{2}$ may be factorized as $G_{2}(r, r)=n^{2}(r)+$ $\left\langle\hat{\psi}_{\downarrow}^{\dagger}(r) \hat{\psi}_{\uparrow}^{\dagger}(r)\right\rangle\left\langle\hat{\psi}_{\uparrow}(r) \hat{\psi}_{\downarrow}(r)\right\rangle$, where the first term is the Hartree term with atom density $n(r)=n_{\uparrow}(r)=n_{\downarrow}(r)$. This term gives rise to a slow two-body photoassociation process as was observed in the high temperature data of Fig. 3. The second term is non-zero only for correlated pairs and is proportional to $|\Delta|^{2}$, the square of the order parameter. In the BCS limit, $|\Delta|^{2} \propto \epsilon_{F}^{2} e^{-\pi /\left(k_{F}|a|\right)}$, whereas in the BEC limit, $|\Delta|^{2} \propto \epsilon_{F}^{2} /\left(k_{F} a\right)$ [17], which is simply proportional to $n(r)$, and produces a rapid onebody loss. In Fig. 5 the data is compared with these functional forms. The fact that the data have the correct dependence on $\left(k_{F} a\right)^{-1}$ in the BEC and BCS limits is suggestive that such an approach has captured the essential physics. Data obtained by starting with higher initial temperatures do not fit the curves given in Fig. 5 


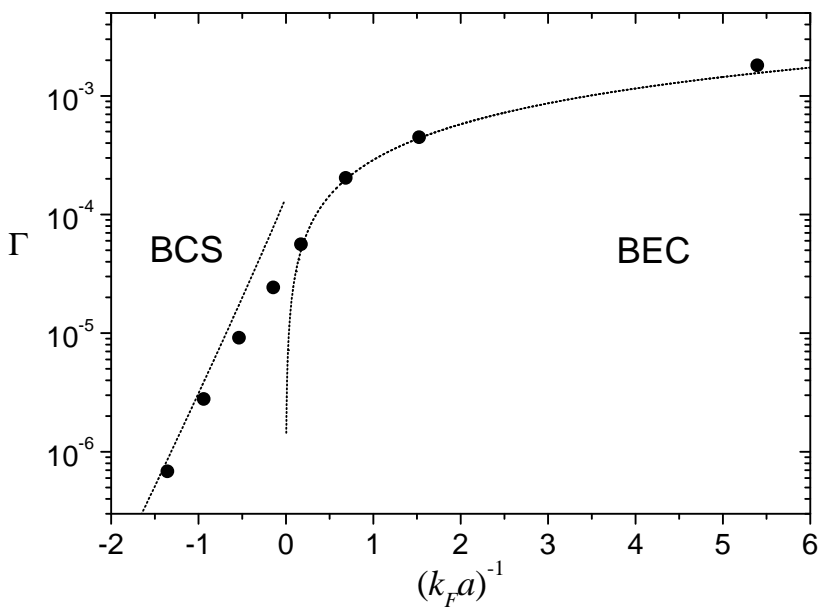

FIG. 5: Comparison of $\Gamma$ with $|\Delta|^{2}$. $\Gamma$ is expressed in units of $\Omega^{2} / \gamma$ making it equivalent to $Z$ plotted in Fig. 4 The dashed lines correspond to evaluations of $|\Delta|^{2}$ in the BCS and the BEC limits integrated over a Thomas-Fermi density profile. They have been scaled by the same factor to give the best fit on the BEC side.

Note that non-condensed pairs [13] will give rise to a similar factorization of $G_{2}$ but it is not expected to have the same dependence on $k_{F} a$. Although the order parameter presented in Fig. 5 comes from a single-channel model, it is the underlying closed-channel part, albeit small, that gives rise to the detectable signal, and indeed to the resonance itself.

We have shown that the singlet closed-channel component of the dressed molecules/pairs is quite small, less than $10^{-3}$, throughout the strongly interacting regime. This result strongly supports the contention of universality for broad resonances. Contrary to expectations from two-body physics, $Z$ does not vanish for fields above resonance, but rather is continuous throughout the resonance regime. Furthermore, the molecular probe distinguishes between the paired and unpaired components of the gas and has enabled measurement of the order parameter in BEC and BCS limits, and throughout the strongly interacting regime.

The authors are grateful to Henk Stoof for extensive interaction, and to Nicolai Nygaard for useful discussions. This work was funded by grants from the NSF, ONR, NASA, and the Welch Foundation.
* Sandia National Laboratories, Livermore, CA 94551

[1] C. A. Regal, M. Greiner, and D. S. Jin, Phys. Rev. Lett. 92, 040403 (2004); M. W. Zwierlein et al., Phys. Rev. Lett. 92, 120403 (2004).

[2] J. Kinast et al., Phys. Rev. Lett. 92, 150402 (2004); M. Bartenstein et al., Phys. Rev. Lett. 92, 203201 (2004); J. Kinast et al., Science 307, 1296 (2005).

[3] For a review of Feshbach physics, see R. A. Duine and H. T. C. Stoof, Phys. Rep. 396, 115 (2004).

[4] R. Combescot, Phys. Rev. Lett. 91, 120401 (2003);G. M. Bruun, Phys. Rev. A 70, 053602 (2004); S. De Palo, M. L. Chiofalo, M. J. Holland, and S. J. J. M. F. Kokkelmans, Phys. Lett. A 327, 490 (2004); R. B. Diener and T.L. Ho, cond-mat/0405174; S. Simonucci, P. Pieri, and G. C. Strinati, Europhys. Lett. 69, 713 (2005); M. H. Szymańska, K. Góral, T. Köhler, and K. Burnett, condmat/0501728.

[5] K. E. Strecker, G. B. Partridge, and R. G. Hulet, Phys. Rev. Lett. 91, 080406 (2003).

[6] M. Houbiers, H. T. C. Stoof, W. I. McAlexander, and R. G. Hulet, Phys. Rev. A 57, R1497 (1998).

[7] M. Bartenstein et al., Phys. Rev. Lett. 94, 103201 (2005).

[8] S. Jochim et al., Science 302, 2101 (2003); M. W. Zwierlein et al., Phys. Rev. Lett. 91, 250401 (2003); T. Bourdel et al., Phys. Rev. Lett. 93, 050401 (2004).

[9] The atomic scattering length $a$ is determined from a coupled channels calculation [6], where we have adjusted our triplet potential slightly to agree with the recently measured resonance location of $834 \mathrm{G}$ 7].

[10] M. Bartenstein et al., Phys. Rev. Lett. 92, 120401 (2004).

[11] I. D. Prodan et al., Phys. Rev. Lett. 91, 080402 (2003).

[12] E. R. I. Abraham, N. W. M. Ritchie, W. I. McAlexander, and R. G. Hulet, J. Chem. Phys. 103, 7773 (1995).

[13] For a review, see Q. Chen, J. Stajic, S. Tan, and K. Levin, cond-mat/0404274.

[14] G. M. Falco and H. T. C. Stoof, cond-mat/0409621.

[15] The exact quantity obtained from the coupled channels calculation is $\left|\left\langle\psi_{v^{\prime}=68}(S=0) \mid \psi_{p}(B)\right\rangle /\left\langle\psi_{v^{\prime}=68}(S=0) \mid \psi_{p}(B=0)\right\rangle\right|^{2}$.

[16] L. D. Carr, G. V. Shlyapnikov, and Y. Castin, Phys. Rev. Lett. 92, 150404 (2004); J. E. Williams, N. Nygaard, and C. W. Clark, New J. Phys. 6, 123 (2004); Q. Chen, J. Stajic, and K. Levin, cond-mat/0411090.

[17] J. R. Engelbrecht, M. Randeria, and C. A. R. Sá de Melo, Phys. Rev. B 55, 15153 (1997). 